\title{
Latent tuberculosis infection in healthcare workers in low- and middle-income countries: an updated systematic review
}

\author{
Lika Apriani (1) $^{1,2,3}$, Susan McAllister ${ }^{3}$, Katrina Sharples ${ }^{3,4}$, \\ Bachti Alisjahbana ${ }^{1,5}$, Rovina Ruslami ${ }^{1,6}$, Philip C. Hill ${ }^{3}$ and Dick Menzies ${ }^{7}$ \\ Affiliations: ${ }^{1}$ TB-HIV Research Centre, Faculty of Medicine, Universitas Padjadjaran, Bandung, Indonesia. \\ ${ }^{2}$ Dept of Public Health, Faculty of Medicine, Universitas Padjadjaran, Bandung, Indonesia. ${ }^{3}$ Centre for \\ International Health, Dept of Preventive and Social Medicine, University of Otago, Dunedin, New Zealand. \\ ${ }^{4}$ Dept of Mathematics and Statistics, University of Otago, Dunedin, New Zealand. ${ }^{5}$ Dept of Internal Medicine, \\ Faculty of Medicine, Universitas Padjadjaran, Bandung, Indonesia. ${ }^{6}$ Dept of Biomedical Sciences, Faculty of \\ Medicine, Universitas Padjadjaran, Bandung, Indonesia. ${ }^{7}$ Montreal Chest Institute, McGill University Montreal, \\ Montreal, QC, Canada.
}

Correspondence: Lika Apriani, TB-HIV Research Centre, Faculty of Medicine, Universitas Padjadjaran, Jl. Prof. Eijkman No. 38, Bandung 40161, Indonesia. E-mail: likaajidgmail.com

@ERSpublications

Healthcare workers in low- and middle-income countries remain at increased risk of latent tuberculosis infection http://ow.ly/M4e730nvI6h

Cite this article as: Apriani L, McAllister S, Sharples K, et al. Latent tuberculosis infection in healthcare workers in low- and middle-income countries: an updated systematic review. Eur Respir J 2019; 53: 1801789 [https://doi.org/10.1183/13993003.01789-2018].

ABSTRACT Healthcare workers (HCWs) are at increased risk of latent tuberculosis (TB) infection (LTBI) and TB disease.

We conducted an updated systematic review of the prevalence and incidence of LTBI in HCWs in lowand middle-income countries (LMICs), associated factors, and infection control practices. We searched MEDLINE, Embase and Web of Science (January 1, 2005-June 20, 2017) for studies published in any language. We obtained pooled estimates using random effects methods and investigated heterogeneity using meta-regression.

85 studies (32630 subjects) were included from 26 LMICs. Prevalence of a positive tuberculin skin test (TST) was $14-98 \%$ (mean 49\%); prevalence of a positive interferon- $\gamma$ release assay (IGRA) was $9-86 \%$ (mean 39\%). Countries with TB incidence $\geqslant 300$ per 100000 had the highest prevalence (TST: pooled estimate 55\%, 95\% CI 41-69\%; IGRA: pooled estimate 56\%, 95\% CI 39-73\%). Annual incidence estimated from the TST was 1-38\% (mean 17\%); annual incidence estimated from the IGRA was 10-30\% (mean $18 \%$ ). The prevalence and incidence of a positive test was associated with years of work, work location, TB contact and job category. Only 15 studies reported on infection control measures in healthcare facilities, with limited implementation.

HCWs in LMICs in high TB incidence settings remain at increased risk of acquiring LTBI. There is an urgent need for robust implementation of infection control measures.

This article has supplementary material available from erj.ersjournals.com

Received: Sept 202018 | Accepted after revision: Jan 172019

This study is registered at PROSPERO with identifier number CRD42017079494.

Copyright OERS 2019 


\section{Introduction}

Healthcare workers (HCWs), especially in high tuberculosis (TB) incidence countries, are at increased risk of latent TB infection (LTBI) and TB disease due to exposure to TB cases and variable implementation of infection control practices [1-3]. World Health Organization (WHO) guidelines (2009) advise regular screening for TB in HCWs and routine reporting [4]. A systematic review by JosHi et al. [1] of 51 studies from low- and middle-income countries (LMICs) published from 1999 to 2005 reported that the prevalence of LTBI (tuberculin skin test (TST) positive) among HCWs ranged from 33\% to 79\%. Reported independent risk factors for LTBI included working in medical wards, participation in procedures such as sputum collection and autopsy, and a history of TB patient contact. The annual incidence of LTBI ranged from $0.5 \%$ to $14.3 \%$. Reported independent risk factors for TST conversion were a higher level of clinical training, nursing occupation and recent exposure to TB [1].

Interferon- $\gamma$ release assays (IGRAs) have emerged as alternative diagnostic tests for LTBI $[5,6]$. They are not subject to sensitisation or boosting [3], but they have much higher rates of largely unexplained test conversions and reversions [3,7]. Studies reporting the prevalence and incidence of LTBI in HCWs in LMICs conducted since the 2006 review by Joshi et al. [1] have used the TST, IGRA or both to diagnose LTBI [8-13]. Although two systematic reviews have been carried out recently, one included only studies using the TST [14] and the other included only studies with a comparison group of non-HCWs [2]. We conducted a comprehensive systematic review of the prevalence and incidence of LTBI in HCWs in LMICs, along with factors associated with LTBI. Including studies that used the TST and/or IGRA, we aimed to describe the prevalence and incidence of LTBI in HCWs in LMICs, and to identify associated risk factors, with a particular emphasis on infection control practices.

\section{Methods}

This systematic review was reported according to PRISMA guidelines [15]. The protocol was registered with the PROSPERO register of systematic reviews (identifier CRD42017079494).

\section{Search strategy}

We searched the MEDLINE, Embase and Web of Science electronic databases for primary studies in any language. The previous systematic review by JosHi et al. [1] was up to December 2005; therefore, our search was limited to the period from January 1, 2005 to June 20, 2017. Since this was an update, we did not wish to include any studies such as those published in 2005 that had been included in the JosHI et al. [1] review. If previously included, they were excluded from our review.

Initially, three broad concepts were used to identify the medical subject headings (MeSH) and key words in the databases: "latent tuberculosis", "healthcare workers" and "occupational exposure". Several key words were used for "latent tuberculosis" ("latent tuberculosis", "tuberculin test", "interferon-gamma release test", "IGRA"), "healthcare workers" ("healthcare worker", "health personnel", "physician", "medical staff", "hospital staff", "nurses", "community health worker", "nursing students", "medical student") and "occupational exposure" ("occupational exposure", "infectious disease transmission", "occupational disease", "nosocomial exposure"). All key words were searched for in the title, abstract and field key words. Both key words and subjects identified in the databases were used together with "OR". The key words for the concepts of "healthcare workers" and "occupational exposure" were used together with "OR", and then these results were combined using "AND" with "latent tuberculosis" to obtain the final result (details of the complete search strategy are provided in supplementary table S1).

In addition, we examined references cited in studies and reviews identified as being potentially relevant. We contacted authors of studies for further information if required.

\section{Study selection}

Two reviewers (L.A. and S.M.) independently screened the citations (title and abstract) identified. We obtained full-text versions of all studies identified by either reviewer as being potentially relevant. The two reviewers independently assessed the full texts for inclusion, using pre-specified criteria. Differences were resolved by consensus.

We included studies that reported data on the prevalence or incidence of LTBI in HCWs. Based on the WHO World Health Report 2006, the term "health workers" was defined as "all people engaged in actions whose primary intent is to enhance health" [16]. There are two broad types of HCWs: those that deliver health services, either personal or non-personal, called "health service providers", and those not engaged in the direct provision of health services, called "health management and support workers" [16]. We included studies that reported any part-time or full-time HCWs. We also included studies that reported data on healthcare students (HCSs), including nursing, medical, paramedical, pharmacy and dental students. 
We included cross-sectional TST and IGRA surveys, cohort studies reporting TST and IGRA conversion rates, and retrospective or prospective studies on the incidence of LTBI.

We excluded: 1) case reports or case series of LTBI, 2) studies with 10 or fewer participants, 3) commentaries, 4) editorials and reviews, 5) letters that did not report original data, 6) studies evaluating the TST or IGRA for treatment monitoring in HCWs (i.e. not diagnostic purposes), 7) for incidence, short-term serial testing studies (within 1 month), 8) non-commercial/in-house IGRAs, 9) TST or IGRA testing in the context of a known nosocomial outbreak or single-point source exposure or contact tracing following TB diagnosis, 10) conference abstracts, and 11) immunological or diagnostic studies with no prevalence or incidence data.

Included studies were conducted in one or more of the 139 countries classified by the World Bank as LMICs, with gross national income per capita less than USD12 475 for the 2017 fiscal year [17].

\section{Data extraction}

The two reviewers independently extracted data from a subset of the studies (20\%). The interrater agreement between the two reviewers was high $\geqslant 95 \%$. Any disagreement was settled by consensus. Subsequently, one reviewer (L.A.) extracted data from the full set of included studies. Data extracted onto a Google Form (www.google.com/forms/about) included: author, country, survey year, TB incidence rate, study and participant type, number of participants, healthcare facility, test used, bacille Calmette-Guérin (BCG) vaccination status, prevalence of LTBI (with 95\% confidence interval), LTBI incidence (with 95\% confidence interval), results of analyses of occupational and non-occupational risk factors, TB infection control measures, demographic data, and other relevant details about HCWs. Definitions for healthcare facility, HCW, LTBI prevalence (defined as test positivity), LTBI incidence (defined as test conversion), including test definitions of IGRA and TST positivity and conversion, and risk factor of exposures are described in the supplementary methods. LTBI prevalence and incidence, or conversion, results were extracted and reported separately for the TST and IGRA, in view of previously published evidence that results of these two tests may be discordant.

\section{Quality assessment}

We assessed the quality of studies based on the Cochrane Group guidelines for observational studies [18], according to five criteria: 1) sampling strategy, 2) response rate $\geqslant 80 \%$ for a cross-sectional study and retention rate $\geqslant 90 \%$ for a cohort study, 3) method of measurement of TB exposure, 4) TST or IGRA performance, and 5) reported results (supplementary table S2). Any disagreement was resolved by consensus.

\section{Data synthesis, meta-analysis and meta-regression}

We evaluated the clinical heterogeneity of the studies, in particular with respect to the type of test used, TB incidence in the general population and type of participants included (HCWs or HCSs). We then evaluated the statistical heterogeneity as assessed by the $I^{2}$ statistic. Since significant heterogeneity $\left(I^{2}>75 \%\right)$ was present, we carried out a stratified analysis and a meta-regression. We used random effects methods to obtained pooled estimates and 95\% confidence intervals [19]. Forest plots were generated for each subgroup analysis. The meta-regression was performed using logistic regression in a generalised estimating equation framework to allow for correlations between prevalence estimates from the same study [20]. Data were analysed using Stata version 14.2 (StataCorp, College Station, TX, USA).

\section{Results}

\section{Description of included studies}

A total of 3537 records and four full-text articles from other systematic reviews were screened (figure 1); 168 full-text articles were assessed for eligibility and 83 articles were excluded. 85 studies met the inclusion criteria, representing 32630 subjects from 26 LMICs [8-13, 21-99] (supplementary table S3). Annual TB incidence rates in countries included ranged from 8 per 100000 in Cuba [25] to 977 per 100000 in South Africa [61].

\section{Prevalence of LTBI measured by TST and IGRA}

The prevalence of a positive TST was measured in 66 studies $[8-13,21,22,24-28,30,31,33-37,39,40$, $42,45-56,58-60,62-66,69-71,73-76,78-82,84-86,88,89,91-95,97]$ and of a positive IGRA in 36 studies $[8-12,22,23,26,34,37,38,41,44,47-49,52,55-57,62,67,68,70,76,78,82,87,89-94,96,98,99]$ (supplementary table S4a and b). The forest plot (figure 2) shows the variability in the estimates of prevalence of a positive TST; estimates ranged from $1 \%$ to $98 \%$ and there was evidence of heterogeneity $(\mathrm{p}<0.0005)$. Based on TST results, the prevalence of LTBI in HCWs ranged from $8 \%$ to $98 \%$ (mean $49 \%$ ) and in HCSs ranged from $1 \%$ to $74 \%$ (mean $32 \%$ ). The prevalence of a positive IGRA ranged from $9 \%$ to 


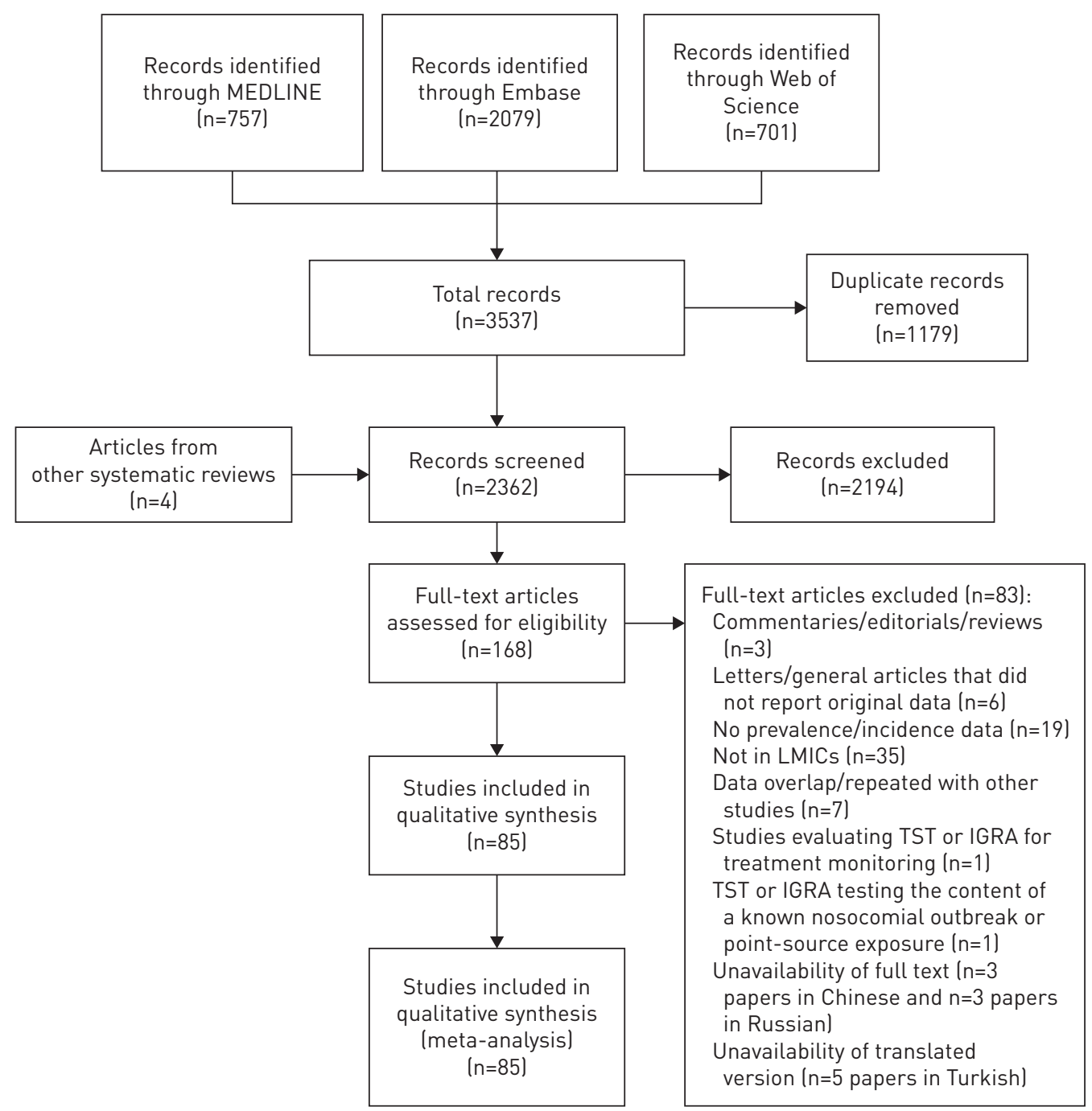

FIGURE 1 Flowchart of literature search. LMICs: low- and middle-income countries; TST: tuberculin skin test; IGRA: interferon- $\gamma$ release assay.

86\% ( $\mathrm{p}$-value for heterogeneity=0.01) (figure 3). The prevalence in HCWs ranged from $9 \%$ to $86 \%$ (mean $39 \%$ ) and in HCSs ranged from $10 \%$ to $44 \%$ (mean $24 \%$ ).

Nurses had the highest prevalence of positive TSTs (pooled estimate 54\%, 95\% CI 46-61\%), followed by physicians (48\%, 95\% CI 30-65\%), allied health professionals (45\%, 95\% CI 35-55\%) and general services (45\%, 95\% CI 30-60\%) (table 1). General services staff had the highest prevalence of positive IGRAs (pooled estimate 60\%, 95\% CI 54-66\%), followed by physicians (35\%, 95\% CI 22-49\%), nurses (34\%, 95\% CI $22-46 \%)$ and allied health professionals (31\%, 95\% CI 23-40\%).

Studies conducted in high TB incidence countries ( $\geqslant 300$ per 100000 population) had the highest prevalence of positive TSTs (pooled estimate 55\%, 95\% CI 41-69\%) and positive IGRAs (56\%, 95\% CI 39-73\%) (table 1 and supplementary figure S1). After stratification by type of participants and then by TB incidence, studies conducted in countries with the lowest TB incidence had the lowest prevalence of LTBI in all types of HCWs and HCSs as measured by the TST or IGRA (supplementary table S4c).

When stratified by study quality, studies with high quality had the lowest prevalence (TST: pooled estimate 41\%, 95\% CI 34-49\%; IGRA: pooled estimate 30\%, 95\% CI 19-41\%) compared with medium- and low-quality studies (table 1). 26 studies investigated the prevalence of LTBI using both the TST and IGRA $[8-12,22,26,34,37,47-49,52,55,56,62,70,76,78,80,82,89,91-94]$ : the prevalence of LTBI measured by the TST was higher (pooled estimate 52\%, 95\% CI 41-62\%) compared with the IGRA (38\%, 95\% CI $38-52 \%$ ) (supplementary figure S2).

For comparison with the review by JosHi et al. [1], we restricted our analysis to studies from five countries (Brazil, India, South Africa, Thailand and Turkey) included in that review (six studies [100-105]) and our 
First author [ref.]

TST: HCWS

AdAms, 2015 [12]

Agara, 2015 [21]

BABAYIGIT, 2014 [22]

BeLo, 2017 [24]

BORROTO, 2011 [25]

BozKanat, 2016 [9]

Caglayan, 2011 [26]

DA Costa, 2006 [33]

De Miranda, 2012 [35]

DE OLIVEIRA, 2007 [36]

De Souza, 2014 [37]

EL-SoKKARY, 2015 [10]

ENCINALES, 2010 [40]

Franco, 2006 [42]

GUTIERREZ, 2012 [45]

$\mathrm{HE}, 2010$ [46]

$\mathrm{HE}, 2012$ [47]

$\mathrm{HE}, 2015$ [48]

Hefzy, 2016 [49]

ISLAM, 2014 [51]

KARGI, 2017 [52]

KIERTIBURANAKUL, 2012 [54]

KHAWCHAROENPORN, 2016 [11]

LI-LAN, 2013 [55]

LIEN, 2009 [56]

LOPES, 2008 [58]

MIRTSKHULAVA, 2008 [62]

MoREIRA, 2010 [63]

Mostafavi, 2016 [8]

Munisamy, 2017 [64]

NASEHI, 2017 [65]

NIKOKAR, 2010 [66]

Ozdemir, 2006 [69]

OzDemir, 2007 [70]

Ozsoy, 2010 [71]

PoWELL, 2011 [74]

RABAHI, 2007 [75]

RAFIZA, 2011 [76]

Ratnatunga, 2015 [78]

Rodrigues, 2009 [79]

Rogerio, 2015 [81]

RutANGA, 2015 [13]

SALMANZADEH, 2016 [82]

SAWHNEY, 2015 [84]

SeVEro, 2011 [85]

SHARIFI-Mood, 2006 [86]

TAHERI, 2013 [88]

TAleBi-TAher, 2011 [89]

TOPIC, 2009 [91]

van RIE, 2013 [92]

WEI, 2013 [93]

YALCIN, 2005 [95

Zнои, 2014 [97]

Subtotal $\left(I^{2}=98.4 \%, p=0.00\right)$

TST: HCSS

Calixto-Aguilar, 2016 [27]

CHRISTOPHER, 2010 [28]

Chung-Delgado, 2012 [30]

Corbett, 2007 [31]

Dagnew, 2012 [34]

EMADI-KoochaK, 2009 [39]

НонмUтн, 2006 [50]

KHAWCHAROENPORN, 2009 [53]

Kiertiburanakul, 2012 [54]

Lou, 2015 [59]

MACIEL, 2007 [60]

Perez-Lu, 2013 [73]

RogerIo, 2013 [80]

VAN RIE, 2013 [92]

Subtotal $\left(\left.\right|^{2}=99.7 \%, p=0.00\right)$

TST: HCWs and HCSs

WHITAKER, 2013 [94]

Heterogeneity between groups: $p=0.000$

Overall $\left(/^{2}=96.69 \%, p=0.00\right)$

0.00
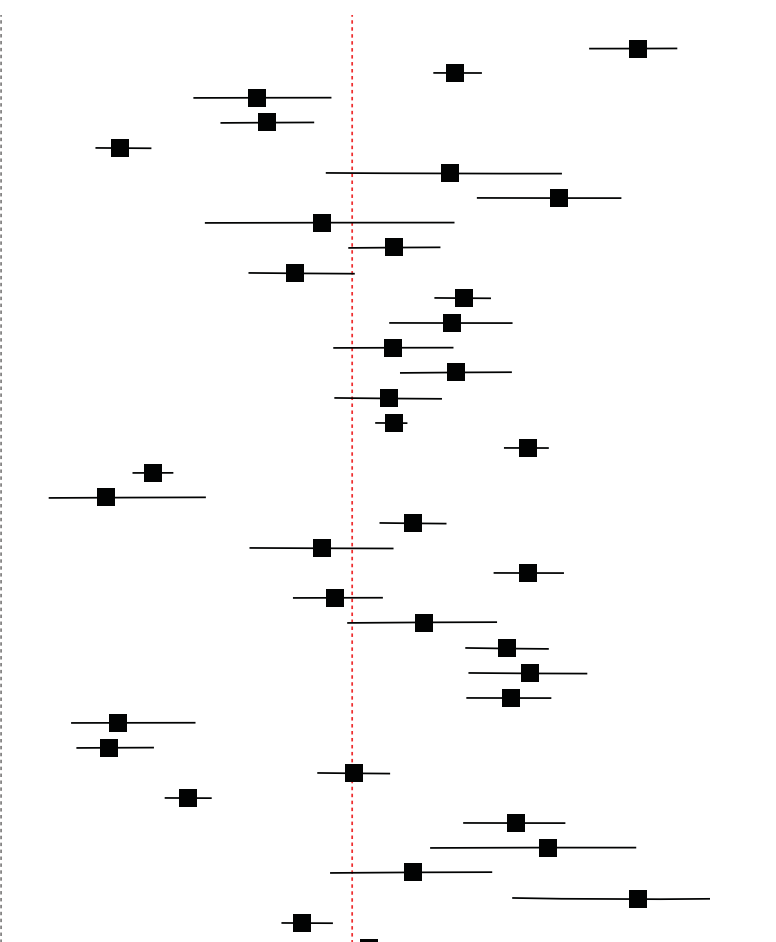

$0.84(0.77-0.89$ $0.59(0.55-0.63)$

$0.33(0.25-0.43)$

$0.34(0.28-0.41)$

$0.15(0.12-0.20)$

$0.59(0.42-0.74)$

$0.73(0.62-0.82)$

$0.42(0.26-0.59)$

$0.51(0.45-0.58)$

$0.39(0.32-0.46)$

$0.61(0.57-0.64)$

$0.59(0.51-0.67)$

$0.51(0.43-0.59)$

$0.60(0.52-0.67)$

$0.51(0.44-0.58)$

$0.51(0.49-0.53)$

$0.69(0.66-0.72)$

$0.20(0.17-0.22)$

$0.14(0.06-0.27)$

$0.54(0.49-0.58)$

$0.42(0.33-0.52)$

$0.69(0.64-0.74)$

$0.44(0.38-0.50)$

$0.55(0.45-0.65)$

$0.66(0.61-0.72)$

$0.70(0.61-0.77)$

$0.67(0.61-0.72)$

$0.15(0.09-0.25)$

$0.14(0.10-0.20)$

$0.46(0.41-0.51)$

$0.24(0.21-0.28)$

$0.68(0.61-0.74)$

$0.72(0.56-0.83)$

$0.54(0.43-0.65)$

$0.84(0.67-0.93)$

$0.40(0.37-0.43)$

$0.48(0.44-0.53)$

$0.59(0.49-0.68)$

$0.47(0.32-0.63)$

$0.27(0.14-0.44)$

$0.59(0.53-0.64)$

$0.62[0.59-0.65]$

$0.36(0.26-0.46)$

$0.20(0.13-0.29)$

$0.47(0.35-0.60)$

$0.55(0.45-0.64)$

$0.08(0.04-0.15)$

$0.52(0.45-0.59)$

$0.63(0.50-0.75)$

$0.57(0.48-0.65)$

$0.98(0.92-0.99)$

$0.24(0.16-0.33)$

$0.44(0.40-0.48)$

$0.49(0.43-0.55)$

0.07 (0.05-0.09)

$0.50(0.46-0.55)$

$0.14(0.12-0.18)$

$0.52(0.47-0.57)$

$0.47(0.38-0.55)$

$0.07(0.04-0.10)$

$0.21(0.18-0.24)$

$0.74(0.67-0.80)$

$0.65(0.62-0.68)$

$0.45(0.39-0.51)$

$0.19(0.16-0.22)$

0.01 (0.01-0.01)

$0.24(0.19-0.30)$

$0.27(0.18-0.37)$

$0.32(0.20-0.45)$

$0.63(0.57-0.68)$

$0.46(0.38-0.54)$

FIGURE 2 Forest plot for the prevalence of latent tuberculosis infection in healthcare workers (HCWs) and healthcare students (HCSs) measured by the tuberculin skin test (TST). 
First author [ref.]

IGRA: HCWs

AdAMs, 2015 [12]

BABAYIGIT, 2014 [22]

Bozkanat, 2016 [9]

Caglayan, 2011 [26]

De Souza, 2014 [37]

DRoBNIEWSKI, 2007 [38]

EL-SOKKARY, 2015 [10]

Escombe, 2010 [41]

GUPTA, 2015 [44]

$\mathrm{HE}, 2012$ [47]

$\mathrm{HE}, 2015$ [48]

HeFzY, 2016 [49]

KARGI, 2017 [52]

KHAWCHAROENPORN, 2016 [11]

LI-FAN, 2013 [55]

LIEN, 2009 [56]

LIU, 2015 [57]

MiRTSKHULAVA, 2008 [62]

Mostafavi, 2016 [8]

NikoLova, 2013 [67]

NoNGHANPHITHAK, 2016 [68]

OZdemIR, 2007 [70]

RAFIZA, 2011 [76]

RatNatunga, 2015 [78]

Salmanzadeh, 2016 [82]

SIDDIOI, 2012 [87]

TALEBI-TAHER, 2011 [89]

THuong, 2016 [90]

ToPIC, 2009 [91]

VAN REI, 2013 [92]

WEI, 2013 [93]

ZHANG, 2013 [96

ZHu, 2014 [98]

Subtotal $\left(\left.\right|^{2}=98.5 \%, p=0.00\right)$

IGRA: HCSS

DAGNEW, 2012 [34]

DROBNIEWSKI, 2007 [38]

VAN REI, 2013 [92]

ZWERLING, 2013 [99]

Subtotal $\left(I^{2}=94.8 \%, p=0.00\right)$

IGRA: HCWs and HCSS

WHITAKER, 2013 [94]

Heterogeneity between groups: $p=0.011$ Overall $\left(\ell^{2}=96.46 \%, p=0.00\right)$
Effect size $(95 \% \mathrm{CI})$

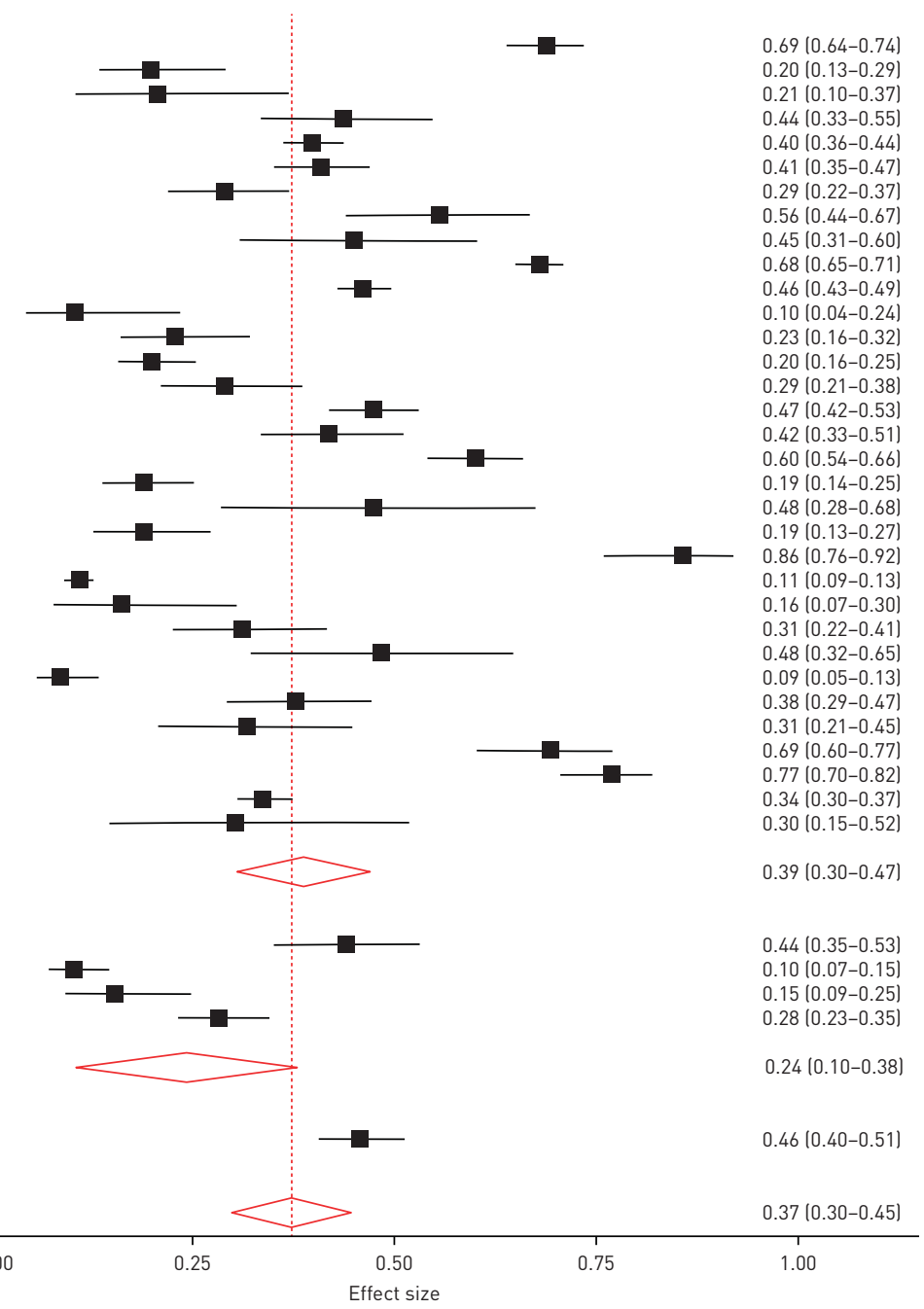

FIGURE 3 Forest plot for the prevalence of latent tuberculosis infection in healthcare workers (HCWs) and healthcare students (HCSs) measured by the interferon- $\gamma$ release assay (IGRA).

review (23 studies $[9,11,12,22,26,33,35-37,42,52,54,58,63,69-71,75,79,81,84,92,95])$. The LTBI prevalence measured by the TST in HCWs in our review was slightly lower (pooled estimate 51\%, 95\% CI 44-58\%) than Joshi et al.'s [1] estimate (55\%, 95\% CI 44-65\%) (supplementary figure S3). Using the same approach in HCSs, when restricted to four countries (Brazil, India, Iran and Uganda), the prevalence of LTBI was higher in five studies from our review $[28,39,59,60,80]$ (pooled estimate $29 \%$, 95\% CI 13-45\%) than seven studies from JosHi et al.'s [1] review [103, 106-111] (24\%, 95\% CI 14-34\%) (supplementary figure S4).

We fitted a meta-regression model using all studies, which provided prevalence estimates (75 studies and 164 prevalence estimates). Studies conducted in high annual TB incidence countries ( $\geqslant 300$ and 200-299 per 100000 population) were statistically significantly associated with LTBI prevalence (OR 8.4, p $<0.001$ and $2.9, \mathrm{p}=0.040$, respectively). HCSs had a statistically significantly lower risk of LTBI (OR $0.3, \mathrm{p}=0.003$ ) (supplementary table S4d).

Risk factors associated with LTBI prevalence

28 studies reported factors associated with a positive TST $[8,13,21,24,25,28,30,34,36,37,46-48,52-$ $55,58,59,62,64,65,74,81,85,92,94,97$ ] (supplementary table S5). In table 2 we provide a summary of risk factors examined and those found statistically significant and not significant. Estimates are not provided due to the potential bias from selective reporting of statistically significant results. Among HCWs, occupational risk factors of more years of work [13, 21, 37, 46, 47, 58, 62, 97] and work location [46, 64, 81, 97] were statistically significantly associated with LTBI prevalence. In some studies, job category $[64,65]$ and contact with a TB patient $[21,25]$ were independent occupational risk factors. 
TABLE 1 Prevalence of latent tuberculosis (TB) infection (LTBI) measured by the tuberculin skin test (TST) and interferon- $\gamma$ release assay (IGRA): differences by study quality

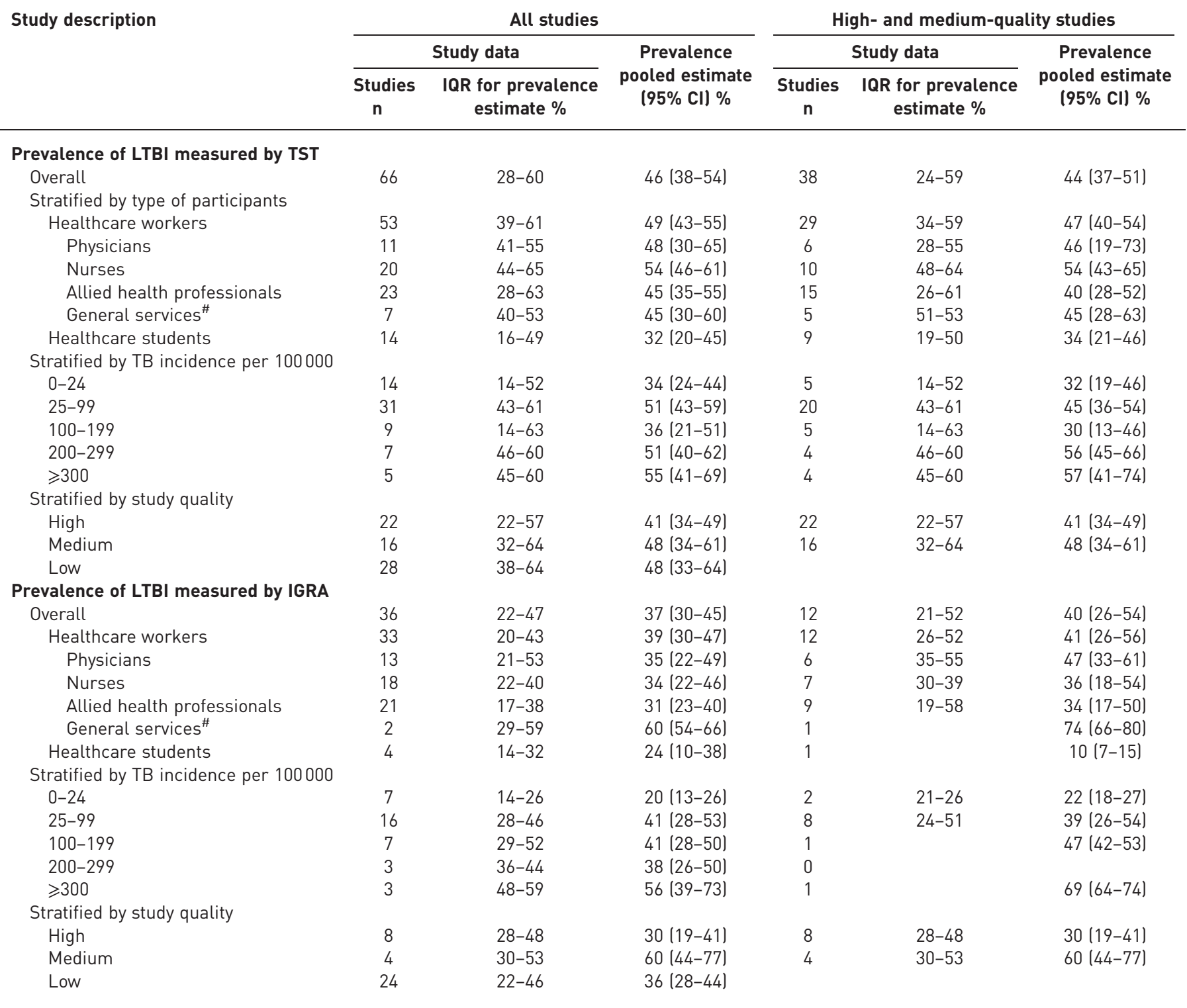

": general services included cleaners, drivers and housekeepers.

In HCSs, years spent in healthcare after entry to a clinical programme [28] and contact with a TB patient [59] were independent risk factors. Male sex [21, 47, 54, 74], age [37, 46, 74, 94], BCG scar/history of BCG vaccination $[37,46,48,54]$, education level $[65,81]$, household contact with TB $[47,81]$, smoking status [36, 48], chronic disease [64, 65], immunosuppression [24] and diabetes mellitus [64] were independent non-occupational risk factors in HCWs. Similarly in HCSs, male sex [54, 59], BCG scar/history of BCG vaccination [53, 54], older age [34], TB knowledge [34] and course of study [59] were associated with a higher LTBI prevalence.

21 studies reported risk factors associated with higher prevalence of LTBI determined by the IGRA $[8,10$, $22,34,37,38,47,48,52,55,56,62,68,70,76,90,92-94,96,99]$ (supplementary table S5). In summary (table 2), among HCWs, a positive IGRA was associated with more years of work [10, 22, 37, 47, 48, 62, $68,96]$, job category $[10,68,76,93,96]$, contact with a TB patient $[55,68]$, type of facility $[22,56]$, work location [38], contact with a co-worker with TB [47] and average daily time with direct patient contact in the preceding year [47]. No occupational factors were significantly associated with a positive IGRA in HCSs. Non-occupational factors significantly associated with a positive IGRA in HCWs included older age 
TABLE 2 Summary of risk factors associated with the prevalence of latent tuberculosis (TB) infection measured by the tuberculin skin test (TST) or interferon- $\gamma$ release assay (IGRA)

\begin{tabular}{|c|c|c|c|c|c|c|c|}
\hline \multirow[t]{2}{*}{ Factors } & \multicolumn{3}{|c|}{ Occupational } & \multirow[t]{2}{*}{ Factors } & \multicolumn{3}{|c|}{ Non-occupational } \\
\hline & $\begin{array}{c}\text { Studies } \\
\text { assessed } \\
n\end{array}$ & $\begin{array}{c}\text { Studies showing } \\
\text { statistically } \\
\text { significant }(p<0.05 \text { ) } \\
\text { association } n \text { [refs] }\end{array}$ & $\begin{array}{l}\text { Studies showing no } \\
\text { statistically significant } \\
\text { association n [refs] }\end{array}$ & & $\begin{array}{c}\text { Studies } \\
\text { assessed } \\
\mathbf{n}\end{array}$ & $\begin{array}{c}\text { Studies showing } \\
\text { statistically } \\
\text { significant }(p<0.05) \\
\text { association } n \text { [refs] }\end{array}$ & $\begin{array}{l}\text { Studies showing no } \\
\text { statistically significant } \\
\text { association n [refs] }\end{array}$ \\
\hline \multicolumn{8}{|c|}{ Healthcare workers (measured by TST) } \\
\hline Years of work & 16 & $\begin{array}{c}8[13,21,37,46,47 \\
58,62,97]\end{array}$ & $\begin{array}{c}8[8,24,48,55,64,65 \\
81,85]\end{array}$ & Male sex & 20 & $4[21,47,54,74]$ & $\begin{array}{c}16[8,13,24,25,36,37 \\
46,48,52,62,64,65 \\
81,85,92,97]\end{array}$ \\
\hline Work location & 9 & $4[46,64,81,97]$ & $5[13,21,37,74,85]$ & Age & 18 & $4[37,47,74,94]$ & $\begin{array}{c}14[8,24,36,47,48,52 \\
54,62,64,65,81,85 \\
92,97]\end{array}$ \\
\hline Job category & 13 & $2[64,65]$ & $\begin{array}{c}11[8,13,21,24,37,46 \\
55,62,74,85,92]\end{array}$ & BCG scar/vaccination & 13 & $4[37,46,48,54]$ & $\begin{array}{c}9[8,24,25,47,52,62, \\
81,85,97]\end{array}$ \\
\hline Contact with a TB patient & 7 & $2[21,25]$ & $5[8,24,55,62,65]$ & Education level & 8 & $2[65,81]$ & $6[8,46-48,62,97]$ \\
\hline Type of facility & 4 & 1 [97] & $3[13,21,47]$ & Household contact with TB & 5 & $2[47,81]$ & $3[24,37,92]$ \\
\hline $\begin{array}{c}\text { Ever had co-workers with } \\
\text { TB }\end{array}$ & 2 & $1[47]$ & $1[24]$ & Smoking status & 9 & $2[36,48]$ & $\begin{array}{c}7[24,37,46,47,65,85, \\
97]\end{array}$ \\
\hline $\begin{array}{l}\text { Cared for a TB patient in } \\
\text { last year }\end{array}$ & 1 & $1[47]$ & & Chronic disease & 2 & $2[64,65]$ & \\
\hline $\begin{array}{l}\text { Last professional contact } \\
\text { with pulmonary TB } \\
\text { patients }\end{array}$ & 1 & $1[58]$ & & Immunosuppression & 2 & $1[24]$ & $1[81]$ \\
\hline $\begin{array}{c}\text { Had TB infection control or } \\
\text { TB training }\end{array}$ & 3 & $1[47]$ & $2[81,97]$ & Diabetes mellitus & 1 & $1[64]$ & \\
\hline $\begin{array}{c}\text { Aware of TB infection } \\
\text { control guidelines }\end{array}$ & 1 & $1[47]$ & & & & & \\
\hline \multicolumn{8}{|c|}{ Healthcare students (measured by TST) } \\
\hline $\begin{array}{l}\text { Time (years) spent in } \\
\text { healthcare work after } \\
\text { entry }\end{array}$ & 2 & $1[28]$ & $1[34]$ & Male sex & 6 & $2[54,59]$ & $4[28,30,53,92]$ \\
\hline \multirow[t]{4}{*}{ Contact with a TB patient } & 3 & 1 [59] & $2[28,53]$ & BCG scar/vaccination & 4 & $2[53,54]$ & $2[28,34]$ \\
\hline & & & & Age & 7 & $1[34]$ & $6[28,30,53,54,59,92]$ \\
\hline & & & & TB knowledge & 2 & $1[92]$ & 1 [59] \\
\hline & & & & Course type & 1 & $1[59]$ & \\
\hline
\end{tabular}




\begin{tabular}{|c|c|c|c|c|c|c|c|}
\hline \multirow[t]{2}{*}{ Factors } & \multicolumn{3}{|c|}{ Occupational } & \multirow[t]{2}{*}{ Factors } & \multicolumn{3}{|c|}{ Non-occupational } \\
\hline & $\begin{array}{c}\text { Studies } \\
\text { assessed } \\
\mathbf{n}\end{array}$ & $\begin{array}{c}\text { Studies showing } \\
\text { statistically } \\
\text { significant ( } p<0.05 \text { ) } \\
\text { association } n \text { [refs] }\end{array}$ & $\begin{array}{l}\text { Studies showing no } \\
\text { statistically significant } \\
\text { association } n \text { [refs] }\end{array}$ & & $\begin{array}{c}\text { Studies } \\
\text { assessed } \\
\mathbf{n}\end{array}$ & $\begin{array}{c}\text { Studies showing } \\
\text { statistically } \\
\text { significant }(p<0.05 \text { ) } \\
\text { association } n \text { [refs] }\end{array}$ & $\begin{array}{l}\text { Studies showing no } \\
\text { statistically significant } \\
\text { association } n \text { [refs] }\end{array}$ \\
\hline \multicolumn{8}{|c|}{ Healthcare workers (measured by IGRA) ${ }^{+}$} \\
\hline Years of work & 16 & $\begin{array}{c}8[10,22,37,47,48 \\
62,68,96]\end{array}$ & $\begin{array}{c}8[8,38,52,55,56,70 \\
76,93]\end{array}$ & Age & 15 & $5[47,62,68,76,93]$ & $\begin{array}{c}10[10,37,38,48,52 \\
55,56,90,92,96]\end{array}$ \\
\hline Job category & 13 & $5[10,68,76,93,96]$ & $\begin{array}{c}8[8,37,52,55,56,62 \\
90,92]\end{array}$ & Male sex & 14 & $4[37,48,52,76]$ & $\begin{array}{c}10[10,38,47,55,56, \\
62,68,90,92,96]\end{array}$ \\
\hline Contact with a TB patient & 4 & $2[55,68]$ & $2[8,62]$ & Household contact with TB & 5 & $3[10,76,96]$ & $2[37,92]$ \\
\hline Type of facility & 3 & $2[22,56]$ & $1[47]$ & BCG scar/vaccination & 9 & $2[37,47]$ & $\begin{array}{c}7[10,48,52,55,62,68, \\
93]\end{array}$ \\
\hline Work location & 5 & $1[38]$ & $4[37,76,90,96]$ & Education level & 7 & $2[48,56]$ & $5[47,62,68,76,96]$ \\
\hline $\begin{array}{c}\text { Ever had co-workers with } \\
\text { TB }\end{array}$ & 1 & $1[47]$ & & Body mass index & 4 & $2[56,90]$ & $2[68,76]$ \\
\hline \multirow[t]{2}{*}{$\begin{array}{l}\text { Average daily time in } \\
\text { patient last year }\end{array}$} & 1 & $1[47]$ & & Granulysin concentration & 1 & $1[90]$ & \\
\hline & & & & Diabetes mellitus & 1 & $1[10]$ & \\
\hline \multicolumn{8}{|c|}{ Healthcare workers (measured by IGRA) } \\
\hline & & & & Age & 2 & 1 [34] & $1[92]$ \\
\hline & & & & Khat consumption ${ }^{f}$ & 1 & $1[34]$ & \\
\hline
\end{tabular}

BCG: bacille Calmette-Guérin. Factors assessed in studies that were not found to be significantly significant: : HIV status [13, 21, 92], alcohol consumption [36, 37, 97], income [46, 48, 97], TB knowledge score [92]; ": smoking status [30, 59], alcohol consumption [30, 59], body mass index [30, 34], HIV status [59], education [28], income [28], religion [28], household contact with TB [92]; ${ }^{+}$: cared for a TB patient in last year [47], had TB infection control training [47], mask use [56], smoking status [10, 37, 47, 48], income [48, 76], comorbidity [37, 76], alcohol consumption [37], hepatitis C virus [10], TB knowledge score [92]; §: number of days spent working on medical wards [99], religion [34], BCG scar/vaccination [34], body mass index [34], male sex [92], HIV [92], household contact with TB [92], TB knowledge score [92]; ${ }^{f}$ : khat (Catha edulis is plant grown commonly in the horn of Africa; the leaves of khat are chewed by people for its stimulant action). 
First author [ref.]

TST: HCWs

ADAMS, 2015 [12]

BORROTO, 2011 [25]

De MiRANDA, 2012 [35]

$\mathrm{HE}, 2015$ [48]

LOPES, 2008 [58]

MoReIRA, 2010 [63]

RABAHI, 2007 [75]

SAWANYAWISUTH, 2012 [83]

Subtotal $\left(\left.\right|^{2}=96.6 \%, p=0.00\right)$

TST: HCS

Calixto-Aguilar, 2016 [27]

ChRISTOPHER, 2011 [29]

Chung-Delgado, 2012 [30]

EMADI-KOOCHAK, 2009 [39]

PAl, 2006 [72]

Subtotal $\left(I^{2}=53.9 \%, p=0.07\right)$

TST: HCWs and HCSs

GonZALEZ, 2011 [43]

Kiertiburanakul, 2012 [54]

Subtotal $\left({ }^{2}=N A, p=N A\right)$

Heterogeneity between groups: $p=0.009$ Overall $\left(l^{2}=94.53 \%, p=0.00\right)$

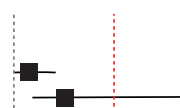

-

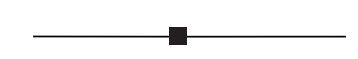

Effect size $(95 \% \mathrm{CI})$

$0.38(0.24-0.55)$ $0.01(0.00-0.04)$ $0.05(0.01-0.17)$ $0.11(0.09-0.15)$ $0.22(0.11-0.39)$

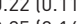
$0.25(0.16-0.37)$ $0.21(0.15-0.29)$ $0.20(0.17-0.22)$

$0.17(0.09-0.24)$

$0.06(0.04-0.07)$ $0.08(0.05-0.13)$ $0.05(0.03-0.07)$

$0.04(0.03-0.06)$

$0.03(0.01-0.06)$

$0.05(0.03-0.06)$

$0.04(0.03-0.06)$ $0.11(0.09-0.15)$

$0.06(0.04-0.07)$

$0.10(0.07-0.13)$

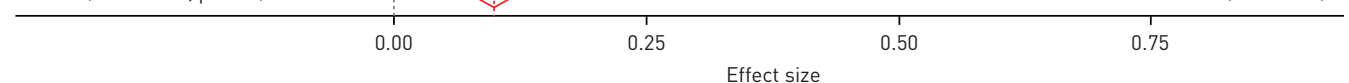

FIGURE 4 Forest plot for the incidence of latent tuberculosis infection in healthcare workers (HCWs) and healthcare students (HCSs) measured by the tuberculin skin test (TST). NA: not applicable.

$[47,62,68,76,93]$, male sex $[37,48,52,76]$, household contact $[10,76,96]$, BCG scar/vaccination $[37,47]$, level of education $[48,56]$, body mass index (BMI) [56, 90], granulysin concentration [90] and diabetes mellitus [10]. In HCSs, older age and khat consumption were the only significant non-occupational factors [34]. Factors assessed in studies that were not found to be statistically significant are listed in the footnotes to table 2 .

\section{Incidence of LTBI measured by TST and IGRA conversion}

The annual LTBI incidence (defined as test conversion after 1 year) was reported in 15 studies using the TST $[12,25,27,29,30,35,39,43,48,54,58,63,72,75,83]$ and five studies using the IGRA [12, 41, 48, $72,77]$ (supplementary table S6a and b). The forest plot (figure 4) shows the variability in the estimates of incidence of LTBI using the TST; estimates ranged from $1 \%$ to $38 \%$ with evidence of significant heterogeneity $(\mathrm{p}<0.009)$. The incidence of LTBI in HCWs ranged from $1 \%$ to $38 \%$ (mean $17 \%)$ and in HCSs ranged from $3 \%$ to $8 \%$ (mean 5\%). For the IGRA, the overall range in incidence estimates was $8-30 \%$ ( $p$-value for heterogeneity=0.016) (figure 5). The incidence in HCWs ranged from $10 \%$ to $30 \%$ (mean 18\%) and in HCSs was $8 \%$ (one study).

Among HCWs, in studies that used the TST, allied health personnel had the highest annual LTBI incidence (pooled estimate 15\%, 95\% CI 3-27\%) followed by physicians (9\%, 95\% CI 0-18\%) and nurses (9\%, 95\% CI 6-12\%). In studies that used the IGRA, physicians had the highest annual incidence (pooled

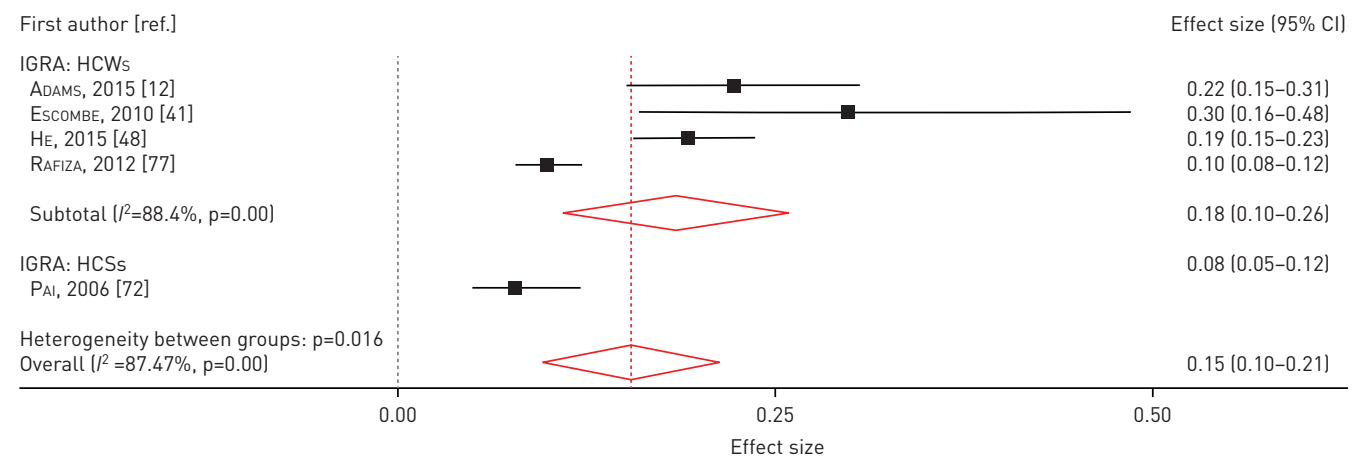

FIGURE 5 Forest plot for the incidence of latent tuberculosis infection in healthcare workers (HCWs) and healthcare students (HCSs) measured by the interferon- $\gamma$ release assay (IGRA). 
estimate $19 \%, 95 \%$ CI $15-23 \%)$ followed by nurses (10\%, 95\% CI $8-13 \%)$ and allied health professionals (10\%, 95\% CI 6-15\%) (table 3).

Studies conducted in countries with TB incidence $\geqslant 300$ per 100000 population had the highest annual incidence of LTBI among HCWs as measured by the TST (pooled estimate 38\%, 95\% CI 24-55\%). In studies that used the IGRA, there was no clear trend, likely due to the low number of studies included $(\mathrm{n}=5)$ (table 3 and supplementary figure S5).

In two studies using both the TST and IGRA [12,48], the annual incidence with the TST was lower (pooled estimate 12\%, 95\% CI 9-15\%) than with the IGRA (20\%, 95\% CI 12-27) (supplementary figure S6). The annual IGRA reversion rate (pooled estimate) was $17 \%$ (95\% CI 7-26\%) in the five studies where it was reported [12, 41, 48, 72, 77] (table 3 and supplementary table S6c).

TABLE 3 Annual incidence of latent tuberculosis (TB) infection (LTBI) measured by the tuberculin skin test (TST) and interferon $-\gamma$ release assay (IGRA): differences by study quality

\begin{tabular}{|c|c|c|c|c|c|c|}
\hline \multirow[t]{3}{*}{ Study description } & \multicolumn{3}{|c|}{ All studies } & \multicolumn{3}{|c|}{ High- and medium-quality studies } \\
\hline & \multicolumn{2}{|r|}{ Study data } & \multirow{2}{*}{$\begin{array}{l}\text { Annual incidence } \\
\text { pooled estimate } \\
{[95 \% \text { CI } \%}\end{array}$} & \multicolumn{2}{|r|}{ Study data } & \multirow{2}{*}{$\begin{array}{c}\text { Annual incidenc } \\
\text { pooled estimate } \\
(95 \% \mathrm{CI}) \%\end{array}$} \\
\hline & $\begin{array}{l}\text { Studies } \\
\mathrm{n}\end{array}$ & $\begin{array}{l}\text { IQR for incidence } \\
\text { estimate } \%\end{array}$ & & $\begin{array}{c}\text { Studies } \\
\mathrm{n}\end{array}$ & $\begin{array}{l}\text { IQR for incidence } \\
\text { estimate } \%\end{array}$ & \\
\hline \multicolumn{7}{|l|}{$\begin{array}{l}\text { Annual incidence of LTBI measured } \\
\text { by TST }\end{array}$} \\
\hline Overall & 15 & $4-21$ & $10(7-13)$ & 11 & $5-22$ & 10 (7-13) \\
\hline \multicolumn{7}{|l|}{ Stratified by type of participants } \\
\hline Healthcare workers & 8 & $10-23$ & $17(9-24)$ & 6 & $14-24$ & $18(9-27)$ \\
\hline Physicians & 3 & $6-14$ & $9(0-18)$ & 1 & & $11(9-15)$ \\
\hline Nurses & 2 & $12-24$ & $9(6-12)$ & 0 & & \\
\hline Allied health professionals & 3 & $11-22$ & $15(3-27)$ & 1 & & $25(16-37)$ \\
\hline General services $\#$ & 0 & & & 0 & & \\
\hline Healthcare students & 5 & $4-6$ & $5(3-6)$ & 4 & $4-6$ & $5(3-6)$ \\
\hline \multicolumn{7}{|c|}{ Stratified by TB incidence per 100000} \\
\hline $0-24$ & 3 & $3-4$ & $3(1-5)$ & 1 & & $1(0-4)$ \\
\hline $25-99$ & 5 & $11-22$ & $16(9-22)$ & 4 & $19-23$ & $19(11-26)$ \\
\hline $100-199$ & 2 & $5-5$ & $5(4-6)$ & 2 & $5-5$ & $5(4-6)$ \\
\hline $200-299$ & 4 & $7-13$ & $10(2-19)$ & 3 & $5-10$ & $7(2-13)$ \\
\hline$\geqslant 300$ & 1 & & $38(24-55)$ & 1 & & $38(24-55)$ \\
\hline \multicolumn{7}{|l|}{ Stratified by study quality } \\
\hline High & 3 & $5-15$ & $7(3-11)$ & 3 & $5-15$ & $7(3-11)$ \\
\hline Medium & 8 & $7-21$ & $11(7-16)$ & 8 & $7-21$ & $11(7-16)$ \\
\hline Low & 4 & $4-9$ & $8(1-16)$ & & & \\
\hline \multicolumn{7}{|l|}{$\begin{array}{l}\text { Annual incidence of LTBI measured } \\
\text { by IGRA }\end{array}$} \\
\hline Overall & 5 & $10-22$ & $15(10-21)$ & 5 & $10-22$ & $15(10-21)$ \\
\hline \multicolumn{7}{|l|}{ Stratified by type of participants } \\
\hline Healthcare workers & 4 & $10-22$ & $18(10-26)$ & 4 & $10-22$ & $18(10-26)$ \\
\hline Physicians & 1 & & 19 (15-23) & 1 & & $19(15-23)$ \\
\hline Nurses & 1 & & $10(8-13)$ & 1 & & $10(8-13)$ \\
\hline Allied health professionals & 1 & & $10(6-15)$ & 1 & & $10(6-15)$ \\
\hline General services ${ }^{\#}$ & 0 & & & 0 & & \\
\hline Healthcare students & 1 & & $8(5-12)$ & 1 & & $8(5-12)$ \\
\hline \multicolumn{7}{|c|}{ Stratified by TB incidence per 100000} \\
\hline $0-24$ & 0 & & & 0 & & \\
\hline $25-99$ & 2 & $12-17$ & $12(10-14)$ & 2 & & $12(10-14)$ \\
\hline $100-199$ & 1 & & $30(16-48)$ & 1 & & $30(16-48)$ \\
\hline $200-299$ & 1 & & $8(5-12)$ & 1 & & $8(5-12)$ \\
\hline$\geqslant 300$ & 1 & & $22(15-31)$ & 1 & & $22(15-31)$ \\
\hline \multicolumn{7}{|l|}{ Stratified by study quality } \\
\hline High & 0 & & & 0 & & \\
\hline Medium & 5 & $10-22$ & $15(10-21)$ & 5 & $10-22$ & $15(10-21)$ \\
\hline Low & 0 & & & & & \\
\hline IGRA reversion rate (annual) & 5 & $7-21$ & $17(7-26)$ & 5 & $7-21$ & $17(7-26)$ \\
\hline
\end{tabular}

${ }^{\#}$ : general services included cleaners, drivers and housekeepers. 
In studies that used the TST, when stratified by study quality, high-quality studies had the lowest incidence (pooled estimate 7\%, 95\% CI 3-11\%) compared with medium-quality studies (11\%, 95\% CI 7-16\%) and low-quality studies (8\%, 95\% CI 1-16). In studies that used the IGRA, none were assessed as low quality (table 3).

\section{Risk factors associated with the incidence of LTBI}

10 studies reported information on risk factors associated with TST conversion [12, 29-32, 48, 61, 73, 83, 94] (supplementary table S7). In summary (table 4), among HCWs, TST conversion was associated with occupational risk factors including job category [83], contact with TB patient [32], years of work [48] and engaged in counselling a TB patient [12]. In HCSs, days spent caring for pulmonary TB patients [29], involvement in sputum collection [29], ever performed or assisted in sputum collection [29] and career type [73] were independent occupational factors. Non-occupational factors in HCWs included male sex [83]; in HCSs, only older age [73] was associated with TST conversion.

Six cohort studies reported factors associated with IGRA conversion [12, 48, 61, 77, 94, 99] (supplementary table S7). Among HCWs, IGRA conversion was associated with work location [77], engaged in counselling a TB patient [12] and minutes spent on diagnosing one patient [61]. Increasing age was the only non-occupational factor [112]. There were no statistically significant factors found in HCSs studies. Factors assessed in studies that were not found to be statistically significant are listed in the footnotes to table 4 .

\section{Infection control measures in healthcare facilities}

Of the 85 studies included in this review, only 15 reported on infection control measures in healthcare facilities $[13,28,32,35,41,42,46,50,54,58,61,74,78,99,113]$ (summarised in supplementary table S8). None implemented a full programme of $\mathrm{TB}$ infection control measures. Nine studies reported inadequate implementation and no clear information was found in six studies. Five of the 15 studies reported a TB infection control policy $[13,28,32,46,113]$. One study reported on TB infection control training [32]. Implementation of patient triage and management was reported in six studies [13, 28, 32, $46,74,78]$, sputum management in one study [74], staff protection (personal respiratory protection) in four studies [28, 32, 35, 42] and environmental control in four studies [13, 32, 42, 46, 54, 74]. To assess the infection control measures, one study used the WHO guidelines [74] and one used the Centers for Disease Control and Prevention guidelines [58].

\section{Discussion}

In this systematic review we have found a high prevalence and incidence of LTBI in HCWs and HCSs in LMICs measured by the TST or IGRA. Nearly $50 \%$ of HCWs and $32 \%$ of HCSs were found to have a positive TST, while $39 \%$ and almost $25 \%$, respectively, had a positive IGRA. HCWs from countries with an annual TB incidence $\geqslant 300$ per 100000 had the highest prevalence of LTBI, with more than half found to be TST or IGRA positive. The annual incidence of LTBI was $17 \%$ in HCWs and $5 \%$ in HCSs when estimated from serial TSTs, and $18 \%$ and $8 \%$, respectively, when estimated with IGRAs.

Strengths of our study include that we used multiple sources and databases to retrieve relevant studies. Paper selection and data extraction were conducted by two independent reviewers, subgroup analyses were used to accommodate heterogeneity across studies, and results were pooled only when studies were reasonably consistent in their methods. Our study does have some limitations. In the absence of a gold standard for diagnosing LTBI, there is no guarantee that prevalence and incidence estimates of LTBI are accurate. Both the TST and IGRA have several limitations. Due to the complexity of the data, in the final analysis we used the definition of LTBI based on the national guideline where the study was done or the standard definition as per the test manufacturer's recommendation. There was substantial heterogeneity, similar to previously published reviews [1, 14], reflecting the different tests, settings and populations included in the review. Although we stratified the prevalence and incidence estimates, due to the heterogeneity in our results, the pooled averages should be interpreted with caution. Although studies published in non-English languages were eligible, five Turkish papers were not accessible and could not be included in the final analysis [114-118]. We extracted data from their English abstracts and, apart from one study [117], the overall results were similar to those in our review (supplementary table S9). We were not able to conduct meta-analyses of the associations of risk factors with LTBI due to limited data. However, all associations are described in supplementary tables S5 and S7, in which all potential risk factors analysed by the authors in each study are summarised along with details regarding which associations were statistically significant. We did not include non-HCWs or non-HCSs in the analysis. A further limitation was not having general population estimates to compare with. The prevalence of LTBI in HCWs from our review was two times higher than the global LTBI prevalence of $23.0 \%$ estimated by Houben and Dodd [119], although the results of their modelling study may have limited comparability to 
TABLE 4 Summary of risk factors associated with the incidence of latent tuberculosis (TB) infection measured by the tuberculin skin test (TST) or interferon- $\gamma$ release assay (IGRA)

\begin{tabular}{|c|c|c|c|c|c|c|c|}
\hline \multirow[t]{2}{*}{ Factors } & \multicolumn{3}{|c|}{ Occupational } & \multirow[t]{2}{*}{ Factors } & \multicolumn{3}{|c|}{ Non-occupational } \\
\hline & $\begin{array}{c}\text { Studies } \\
\text { assessed } \\
\mathbf{n}\end{array}$ & $\begin{array}{c}\text { Studies showing } \\
\text { statistically } \\
\text { significant ( } p<0.05 \text { ) } \\
\text { association } n \text { [refs] }\end{array}$ & $\begin{array}{l}\text { Studies showing no } \\
\text { statistically significant } \\
\text { association } \mathrm{n} \text { [refs] }\end{array}$ & & $\begin{array}{c}\text { Studies } \\
\text { assessed } \\
\mathbf{n}\end{array}$ & $\begin{array}{c}\text { Studies showing } \\
\text { statistically } \\
\text { significant ( } p<0.05 \text { ) } \\
\text { association } n \text { [refs] }\end{array}$ & $\begin{array}{l}\text { Studies showing no } \\
\text { statistically significant } \\
\text { association } \mathrm{n} \text { [refs] }\end{array}$ \\
\hline \multicolumn{8}{|c|}{ Healthcare workers (measured by TST) ${ }^{\#}$} \\
\hline Job category & 3 & 1 [83] & $2[12,32]$ & Male sex & 3 & 1 [83] & $2[12,32]$ \\
\hline Contact with a TB patient & 1 & $1[32]$ & & & & & \\
\hline Years of work & 1 & $1[48]$ & & & & & \\
\hline $\begin{array}{c}\text { Engaged in counselling a } \\
\text { TB patient }\end{array}$ & 1 & $1[12]$ & & & & & \\
\hline \multicolumn{8}{|c|}{ Healthcare students (measured by TST) ${ }^{\pi}$} \\
\hline $\begin{array}{l}\text { Days spent caring for } \\
\text { pulmonary TB patients }\end{array}$ & 1 & $1[29]$ & & Age & 4 & $1[73]$ & $3[29-31]$ \\
\hline $\begin{array}{l}\text { Ever performed or } \\
\text { assisted in sputum } \\
\text { collection }\end{array}$ & 1 & $1[29]$ & & & & & \\
\hline Careers & 1 & $1[73]$ & & & & & \\
\hline \multicolumn{8}{|c|}{ Healthcare workers (measured by IGRA) ${ }^{+}$} \\
\hline Work location & 1 & $1[77]$ & & & & & \\
\hline $\begin{array}{c}\text { Engaged in counselling a } \\
\text { TB patient }\end{array}$ & 1 & $1[12]$ & & & & & \\
\hline $\begin{array}{l}\text { Minutes spent on } \\
\text { diagnosing one patient } \\
\text { Healthcare students (meas }\end{array}$ & 1 & $1[48]$ & & & & & \\
\hline
\end{tabular}

BCG: bacille Calmette-Guérin. Factors assessed in studies that were not found to be significantly significant: \#: work location [32], BCG scar/vaccination [12, 32, 48, 83], age [48], education [48], smoking status [12, 48], income [48], diabetes mellitus [12], alcohol consumption [12], HIV status [12]; ๆ: direct contact with sputum-positive TB [113], days spent working on isolation ward [113], days spent working on pulmonary ward [113], TB contact history [73], male sex [30, 31, 73], BCG scar/vaccination [31, 113], education [113], smoking status [30], income [113], alcohol consumption [30], HIV status [31], household contact with TB [31], type of students [31], body mass index [73]; ${ }^{+}$: years of work [48, 77], job category [12, 77], male sex [48,77], age [48, 77], smoking status [12, 48], education [48], BCG scar/vaccination [48], income [48], diabetes mellitus [12], alcohol consumption [12], HIV status [12]; §: occupational exposures [99]. 
the results of direct testing in the studies included in this review. UDEN et al. [2] found that HCWs and HCSs had more than two times the risk of LTBI than the general population, consistent with previous reports $[1,3]$. Finally, estimating the prevalence and incidence of TB disease was beyond the scope of this review.

This review adds to the evidence that regardless of whether measured by the TST or IGRA, the prevalence of LTBI in HCWs in LMICs continues to be high, especially in high TB incidence countries. This is in keeping with previously published reports that have shown approximately half of HCWs were positive by the TST $[1,14]$. When measured by the IGRA, our overall LTBI prevalence estimate was lower than that measured by the TST, but still high, and consistent with a previously published review in countries with a low and intermediate incidence of TB [3].

Similarly, our review showed that the overall incidence of LTBI in HCWs continues to be high in LMICs. The annual incidence, however, was higher when estimated using the IGRA compared with the TST, which is similar to a previous review [3]. We also found a high rate of IGRA reversion, consistent with previously published reviews in all countries, irrespective of the national TB incidence rates [3, 7]. The exact cause of the substantial rate of IGRA reversion is unexplained $[3,7]$ and raises concerns about the validity of serial IGRA to estimate new infections. Because of this, the WHO has discouraged the use of the IGRA for serial testing in HCWs in LMICs [112].

For HCSs, the prevalence and incidence of LTBI was lower than that seen in HCWs, and this was observed in studies that used the TST and/or IGRA. This is most likely due to their shorter exposure time compared with other HCWs. They do, however, require attention, similar to that needed for other HCWs, as their risk of LTBI is higher compared with the general population [2], as was shown in a Brazilian study in medical and nursing students where the risk of LTBI was more than three times greater than the general population [60].

Across all studies included in our systematic review, occupational factors found to be significantly associated with LTBI in HCWs and HCSs were those that involved more direct contact with a TB patient or prolonged exposure. This is similar to that reported in previous reviews $[1,14,120]$. Hence, these risk factors, along with the continuing high prevalence and incidence of LTBI in HCWs, are an indication that more needs to be done to ensure these workers and students receive the protection they require. The WHO released guidelines on TB infection control in healthcare settings in 2009 [4], yet in our review only 15 studies reported any TB infection control measures (the inadequacy of which was also reported by JosH et al. [1] and NASREEN et al. [14]). In resource-limited countries, budget constraints may be cited as a reason for limited infection control measures. However, the basic control measures recommended in the 2009 WHO guidelines of early identification, isolation and treatment of those with presumptive TB, as well as open-window/door policies, education and training of HCWs, are all low-cost and effective measures that should be feasible in all settings, and provide important protection for these seemingly forgotten workers. Other measures, e.g. infrastructure modification to ensure appropriate natural ventilation/airflow and provision of personal protective measures, are more costly, but may also contribute towards a reduction of TB infection in HCWs. Moreover, it will be important to measure the effectiveness of implementation of infection control measures through monitoring and routine reporting of the number of HCWs who develop TB disease and infection each year in healthcare facilities.

In conclusion, HCWs in LMICs, especially in high TB incidence settings, continue to have an unacceptably high prevalence and incidence of $\mathrm{TB}$ infection. In resource-limited settings, basic control measures as recommended by the $\mathrm{WHO}$, which are low cost and effective, are rarely implemented. TB programmes in high TB incidence countries must prioritise implementation of infection control measures in healthcare facilities to ensure protection for this highly vulnerable and essential group of HCWs.

Acknowledgements: We thank Zhiyi (Tommy) Lan (McGill University, Montreal, QC, Canada) for technical support during the search strategy and study selection of this review, and Leonardo Nava Jimenez (University of Otago, Dunedin, New Zealand) for help with translating Spanish and Portuguese papers.

Conflict of interest: None declared.

\section{References}

1 Joshi R, Reingold AL, Menzies D, et al. Tuberculosis among health-care workers in low- and middle-income countries: a systematic review. PLoS Med 2006; 3: e494.

2 Uden L, Barber E, Ford N, et al. Risk of tuberculosis infection and disease for health care workers: an updated meta-analysis. Open Forum Infect Dis 2017; 4: ofx137.

3 Zwerling A, van den Hof S, Scholten J, et al. Interferon-gamma release assays for tuberculosis screening of healthcare workers: a systematic review. Thorax 2012; 67: 62-70.

4 World Health Organization. WHO Policy on TB Infection Control in Health-care Facilities, Congregate Settings and Households. Geneva, WHO, 2009. 

of uncertainty and recommendations for research. Ann Intern Med 2007; 146: 340-354.

6 Mazurek G, Jereb J, Vernon A, et al. Updated guidelines for using Interferon Gamma Release Assays to detect Mycobacterium tuberculosis infection - United States, 2010. MMWR Recomm Rep 2010; 59: 1-25.

7 Ringshausen FC, Schablon A, Nienhaus A. Interferon-gamma release assays for the tuberculosis serial testing of health care workers: a systematic review. J Occup Med Toxicol 2012; 7: 6.

8 Mostafavi E, Nasehi M, Hashemi Shahraki A, et al. Comparison of the tuberculin skin test and the QuantiFERON-TB Gold test in detecting latent tuberculosis in health care workers in Iran. Epidemiol Health 2016; 38: e2016032

9 Bozkanat E, Kaya H, Sezer O, et al. Comparison of tuberculin skin test and QuantiFERON-TB Gold In-Tube test for diagnosis of latent tuberculosis infection in health care workers: a cross sectional study. J Pak Med Assoc 2016; 66: 270-274.

10 El-Sokkary RH, Abu-Taleb AM, El-Seifi OS, et al. Assessing the prevalence of latent tuberculosis among health care providers in Zagazig City, Egypt using tuberculin skin test and Quantiferon-TB Gold In-Tube Test. Cent Eur J Public Health 2015; 23: 324-330.

11 Khawcharoenporn T, Apisarnthanarak A, Sangkitporn S, et al. Tuberculin skin test and QuantiFERON-TB Gold In-Tube Test for diagnosing latent tuberculosis infection among Thai healthcare workers. Jpn J Infect Dis 2016; 69: 224-230.

12 Adams S, Ehrlich R, Baatjies R, et al. Incidence of occupational latent tuberculosis infection in South African healthcare workers. Eur Respir J 2015; 45: 1364-1373.

13 Rutanga C, Lowrance DW, Oeltmann JE, et al. Latent tuberculosis infection and associated factors among health care workers in Kigali, Rwanda. PLoS One 2015; 10: e0124485.

14 Nasreen S, Shokoohi M, Malvankar-Mehta MS. Prevalence of latent tuberculosis among health care workers in high burden countries: a systematic review and meta-analysis. PLoS One 2016; 11: 19.

15 Hutton B, Salanti G, Caldwell DM, et al. The PRISMA extension statement for reporting of systematic reviews incorporating network meta-analyses of health care interventions: checklist and explanations. Ann Intern Med 2015; 162: 777-784.

16 World Health Organization. Health Workers: A Global Profile. The World Health Report 2006. Geneva, WHO, 2006.

17 World Bank. World Bank Country and Lending Groups: Country Classification. 2017. https://datahelpdesk. worldbank.org/knowledgebase/articles/906519-world-bank-country-and-lending-groups Date last accessed: February $1,2019$.

18 Higgins J, Green S. Cochrane Handbook for Systematic Reviews of Interventions Version 5.1.0 [Updated March 2011]. London, The Cochrane Collaboration, 2011.

19 Nyaga VN, Arbyn M, Aerts M. Metaprop: a Stata command to perform meta-analysis of binomial data. Arch Public Health 2014; 72: 39.

20 Liang K-Y, Zeger SL. Longitudinal data analysis using generalized linear models. Biometrika 1986; 73: 13-22.

21 Agaya J, Nnadi CD, Odhiambo J, et al. Tuberculosis and latent tuberculosis infection among healthcare workers in Kisumu, Kenya. Trop Med Int Health 2015; 20: 1797-1804

22 Babayigit C, Ozer B, Inandi T, et al. Performance of QuantiFERON-TB Gold In-Tube test and tuberculin skin test for diagnosis of latent tuberculosis infection in BCG vaccinated health care workers. Med Sci Monit 2014; 20: 521-529.

23 Bandyopadhyay M, Bhakta A, Chakrabarty S, et al. Clinical and bacteriological correlates of whole blood interferon gamma (IFN-gamma) in newly detected cases of pulmonary TB. Asian Pac J Trop Med 2010; 3: 224-231.

24 Belo C, Naidoo S. Prevalence and risk factors for latent tuberculosis infection among healthcare workers in Nampula Central Hospital, Mozambique. BMC Infect Dis 2017; 17: 408.

25 Borroto S, Gamez D, Diaz D, et al. Latent tuberculosis infection among health care workers at a general hospital in Santiago de Cuba. Int J Tuberc Lung Dis 2011; 15: 1510-1514.

26 Caglayan V, Ak O, Dabak G, et al. Comparison of tuberculin skin testing and QuantiFERON-TB Gold In-Tube test in health care workers. Tuberk Toraks 2011; 59: 43-47.

27 Calixto-Aguilar LS, Manrique-Zegarra M, Gotuzzo-Herencia E, et al. Conductas frente al viraje de la prueba de tuberculina en estudiantes de medicina de una universidad de Lima, Peru. [Behaviors in response to the tuberculin skin test conversion in medical students from a university in Lima, Peru.] Rev Peru Med Exp Salud Publica 2016; 33: 283-287.

28 Christopher DJ, Daley P, Armstrong L, et al. Tuberculosis infection among young nursing trainees in South India. PLoS One 2010; 5: e10408.

29 Christopher DJ, James P, Michael JS, et al. Prevalence of LTBI using IGRA and TST in a cohort of health professional trainees from India. Eur Respir J 2011; 38: Suppl. 55, p2652.

30 Chung-Delgado K, Guillen-Bravo S, Navarro-Huaman L, et al. Estudiantes de medicina en riesgo: prevalencia e incidencia de conversion de PPD. [Medical students at risk: prevalence and incidence of tuberculin skin test conversion.] Rev Chilena Infectol 2012; 29: 375-381.

31 Corbett EL, Muzangwa J, Chaka K, et al. Nursing and community rates of Mycobacterium tuberculosis infection among students in Harare, Zimbabwe. Clin Infect Dis 2007; 44: 317-323.

32 da Costa PA, Trajman A, Mello FC, et al. Administrative measures for preventing Mycobacterium tuberculosis infection among healthcare workers in a teaching hospital in Rio de Janeiro, Brazil. J Hosp Infect 2009; 72: 57-64.

33 Da Costa HCG, Malaspina AC, De Mello FAF, et al. Tuberculosis in a psychiatric hospital in the state of Goias, Brazil. J Bras Pneumol 2006; 32: 566-572.

34 Dagnew AF, Hussein J, Abebe M, et al. Diagnosis of latent tuberculosis infection in healthy young adults in a country with high tuberculosis burden and BCG vaccination at birth. BMC Res Notes 2012; 5: 415

35 De Miranda SS, De Oliveira AC, Santos AX, et al. Positive tuberculin test and risk of infection by Mycobacterium tuberculosis in a tuberculosis clinic settled in an upright building, in Minas Gerais, Brazil. Rev Med Chil 2012; 140: 1022-1027.

36 de Oliveira S, Honner MR, Paniag AM, et al. Prevalence of mycobacterium tuberculosis among professionals in a university hospital, Mato Grosso do Sul, 2004. Rev Lat Am Enfermagem 2007; 15: 1120-1124. 

points of tuberculin skin test to detect latent Mycobacterium tuberculosis infection in primary health care workers. PLoS One 2014; 9: e102773.

38 Drobniewski F, Balabanova Y, Zakamova E, et al. Rates of latent tuberculosis in health care staff in Russia. PLoS Med 2007; 4: e55.

39 Emadi-Koochak H, Rasoulinejad M, Alinaghi SAS, et al. Tuberculin skin test conversion among students during their educational course in medical and pharmacy schools: a multiple cohort study. Tanaffos 2009; 8: 33-36.

40 Encinales L, Zuniga J, Granados-Montiel J, et al. Humoral immunity in tuberculin skin test anergy and its role in high-risk persons exposed to active tuberculosis. Mol Immunol 2010; 47: 1066-1073.

41 Escombe AR, Huaroto L, Ticona E, et al. Tuberculosis transmission risk and infection control in a hospital emergency department in Lima, Peru. Int J Tuberc Lung Dis 2010; 14: 1120-1126.

42 Franco C, Zanetta DMT. Assessing occupational exposure as risk for tuberculous infection at a teaching hospital in Sao Paulo, Brazil. Int J Tuberc Lung Dis 2006; 10: 384-389.

43 Gonzalez CD, Barth MDLA, Araujo GC, et al. Tasas de conversión de tuberculina entre trabajadores de la salud. [Tuberculin conversion rates among health care workers.] Salud Cienc 2011; 18: 157-159.

44 Gupta V, Athavale AU, Natraj G. Comparative study for evaluating T SPOT-TB in analysis between low and high risk subjects - a pilot study. Int J Med Res Health Sci 2015; 4: 360-365.

45 Gutierrez SB, Court JIS, Leru MF, et al. Riesgo de ocurrencia de la tuberculosis en los trabajadores del Hospital Universitario Neumologico Benefico Juridico de La Habana. [Tuberculosis risk assessment in the staff of the National University Pneumologic Hospital of Havana.] Rev Cubana Med Trop 2012; 64: 55-60.

46 He GX, van den Hof S, van der Werf MJ, et al. Infection control and the burden of tuberculosis infection and disease in health care workers in china: a cross-sectional study. BMC Infect Dis 2010; 10: 313.

47 He GX, Wang LX, Chai SJ, et al. Risk factors associated with tuberculosis infection among health care workers in Inner Mongolia, China. Int J Tuberc Lung Dis 2012; 16: 1485-1491.

$48 \mathrm{He}$ GX, Li Y, Zhao F, et al. The prevalence and incidence of latent tuberculosis infection and its associated factors among village doctors in China. PLoS One 2015; 10: 14

49 Hefzy EM, Wegdan AA, Elhefny RA, et al. Predictors of low prevalence of latent tuberculosis infection among Egyptian health care workers at intensive care and bronchoscopy units. GMS Hyg Infect Control 2016; 11 : 9.

50 Hohmuth BA, Yamanija JC, Dayal AS, et al. Latent tuberculosis infection: risks to health care students at a hospital in Lima, Peru. Int J Tuberc Lung Dis 2006; 10: 1146-1151.

51 Islam S. Latent tuberculosis infection among healthcare workers in chest disease hospitals, Bangladesh. Health Sci Bull 2014; 12: 1-7.

52 Kargi A, Ilgazli AH, Yildiz F, et al. Latent tuberculosis infection in healthcare workers at a tertiary care center. Biomed Res India 2017; 28: 657-662.

53 Khawcharoenporn T, Apisarnthanarak A, Thongphubeth $\mathrm{K}$, et al. Tuberculin skin tests among medical students with prior bacille-Calmette Guerin vaccination in a setting with a high prevalence of tuberculosis. Infect Control Hosp Epidemiol 2009; 30: 705-709.

54 Kiertiburanakul S, Suebsing S, Kehachindawat P, et al. Five-year prospective study of tuberculin skin testing among new healthcare personnel at a university hospital in Thailand. J Hosp Infect 2012; 80: 173-175.

55 Li-fan Z, Xiao-qing L, Yao Z, et al. A prospective longitudinal study evaluating a T-cell-based assay for latent tuberculosis infection in health-care workers in a general hospital in Beijing. Chin Med J 2013; 126: $2039-2044$.

56 Lien LT, Hang NT, Kobayashi N, et al. Prevalence and risk factors for tuberculosis infection among hospital workers in Hanoi, Viet Nam. PLoS One 2009; 4: e6798.

57 Liu Y, Ou M, He S, et al. Evaluation of a domestic interferon-gamma release assay for detecting Mycobacterium tuberculosis infection in China. Tuberculosis 2015; 95: 523-526.

58 Lopes LK, Teles SA, Souza AC, et al. Tuberculosis risk among nursing professionals from Central Brazil. Am J Infect Control 2008; 36: 148-151.

59 Lou JK, Okot-Nwang M, Katamba A. Prevalence of positive tuberculin skin test and associated factors among Makerere medical students, Kampala, Uganda. Afr Health Sci 2015; 15: 1247-1255.

60 Maciel ELN, Meireles W, Silva AP, et al. Nosocomial Mycobacterium tuberculosis transmission among healthcare students in a high incidence region, in Vitoria, State of Espirito Santo. Rev Soc Bras Med Trop 2007; 40: 397-399.

61 McCarthy KM, Scott LE, Gous N, et al. High incidence of latent tuberculous infection among South African health workers: an urgent call for action. Int J Tuberc Lung Dis 2015; 19: 647-653.

62 Mirtskhulava V, Kempker R, Shields KL, et al. Prevalence and risk factors for latent tuberculosis infection among health care workers in Georgia. Int J Tuberc Lung Dis 2008; 12: 513-519.

63 Moreira TR, Zandonade E, Maciel EL. Risk of tuberculosis infection among community health agents. Rev Saude Publica 2010; 44: 332-338.

64 Munisamy M, Krishnan K, Selvaratnam G, et al. Not Tb-proof: latent tuberculosis in Kuala Lumpur Hospital health care workers. Occup Med 2017; 67: 224-226.

65 Nasehi M, Hashemi-Shahraki A, Doosti-Irani A, et al. Prevalence of latent tuberculosis infection among tuberculosis laboratory workers in Iran. Epidemiol Health 2017; 39: e2017002.

66 Nikokar I, Dadgran A, Mafozei L. A comparison of two-step tuberculin skin test between health-care workers and nonhospital employees. Iran J Med Sci 2010; 35: 201-204.

67 Nikolova M, Muhtarova M, Drenska R, et al. Peripheral blood CD8 T cell response in different phases of MTB infection. C R Acad Bulg Sci 2013; 66: 587-594.

68 Nonghanphithak D, Reechaipichitkul W, Chaiyasung T, et al. Risk factors for latent tuberculosis infection among health-care workers in Northeastern Thailand. Southeast Asian J Trop Med Public Health 2016; 47: 1198-1208.

69 Ozdemir D, Cesur S, Annakkaya AN, et al. Serum neopterin concentrations in healthy healthcare workers compared with healthy controls and patients with pulmonary tuberculosis. Med Sci Monit 2006; 12: CR521-CR524. Ozdemir D, Annakkaya AN, Tarhan G, et al. Comparison of the tuberculin skin test and the quantiferon test for latent Mycobacterium tuberculosis infections in health care workers in Turkey. Jpn J Infect Dis 2007; 60: 102-105.

71 Ozsoy S, Akar T, Gumus S, et al. The results of tuberculin skin test and the risk of tuberculosis in autopsy workers. Turk Klin J Med Sci 2010; 30: 1876-1883. 
Pai M, Joshi R, Dogra S, et al. Serial testing of health care workers for tuberculosis using interferon-gamma assay. Am J Respir Crit Care Med 2006; 174: 349-355.

Perez-Lu JE, Carcamo CP, Garcia PJ, et al. Tuberculin skin test conversion among health sciences students: a retrospective cohort study. Tuberculosis 2013; 93: 257-262.

Powell K, Han D, Hung NV, et al. Prevalence and risk factors for tuberculosis infection among personnel in two hospitals in Viet Nam. Int J Tuberc Lung Dis 2011; 15: 1643-1649.

Rabahi MF, Junqueira-Kipnis AP, Dos Reis MC, et al. Humoral response to HspX and GlcB to previous and recent infection by Mycobacterium tuberculosis. BMC Infect Dis 2007; 7: 148.

Rafiza S, Rampal KG, Tahir A. Prevalence and risk factors of latent tuberculosis infection among health care workers in Malaysia. BMC Infect Dis 2011; 11: 19.

Rafiza S, Rampal KG. Serial testing of Malaysian health care workers with QuantiFERON-TB Gold In-Tube. Int Tuberc Lung Dis 2012; 16: 163-168.

Ratnatunga CN, Thevanesam V, Nandadeva D, et al. Cross-sectional screening of healthcare workers at a regional chest clinic with an interferon gamma release assay: first report from Sri Lanka. Healthc Infect 2015; 20: 89-94.

Rodrigues PM, Moreira TR, de Moraes AKL, et al. Mycobacterium tuberculosis infection among community health workers involved in TB control. J Bras Pneumol 2009; 35: 351-358.

Rogerio WP, Baraona CMO, do Prado TN, et al. Prevalencia de infeccao latente pelo Mycobacterium tuberculosis entre estudantes da area da saude de uma universidade publica em Vitoria, ES, Brasil. [Prevalence of latent infection of Mycobacterium tuberculosis among healthcare students in a public university in Vitoria, state of Espirito Santo, Brazil.] Cien Saude Colet 2013; 18: 1331-1339.

Rogerio WP, do Prado TN, de Souza FM, et al. Prevalencia e fatores associados a infeccao pelo Mycobacterium tuberculosis entre agentes comunitarios de saude no Brasil, usando-se a prova tuberculinica. [Prevalence of infection with Mycobacterium tuberculosis and associated factors in community health workers in Brazil based on the tuberculin skin test.] Cad Saude Publica 2015; 31: 2199-2210.

Salmanzadeh S, Abbasissifar H, Alavi SM. Comparison study of QuantiFERON test with tuberculin skin testing to diagnose latent tuberculosis infection among nurses working in teaching hospitals of Ahvaz, Iran. Caspian $J$ Intern Med 2016; 7: 82-87.

Sawanyawisuth K, Chaiear N, Sawanyawisuth K, et al. Can job titles be predictors for recent onset latent tuberculosis in health care workers? Asian Biomed 2012; 6: 535-539.

Sawhney N, Mehta S, Singh V, et al. Application of tuberculin skin test in diagnosis of latent tuberculosis: a two year experience in a tertiary care hospital. J Pharm Biomed Sci 2015; 05: 643-649.

Severo KGP, Oliveira JD, Carneiro M, et al. Latent tuberculosis in nursing professionals of a Brazilian hospital. J Occup Med Toxicol 2011; 6: 4.

Sharifi-Mood B, Savadkouhi F, Salehi M, et al. Comparison of ultrasonographic measurement of the tuberculin skin test with the result of manual reading. J Med Sci 2006; 6: 389-392.

Siddiqi UR, Leano PS, Chagan-Yasutan H, et al. Frequent detection of anti-tubercular-glycolipid-IgG and -IgA antibodies in healthcare workers with latent tuberculosis infection in the Philippines. Clin Dev Immunol 2012; 2012: 610707.

Taheri M, Bazrafkan H, Habibagahi M. Determining the latent tuberculosis infection by IFN-gamma ELISPOT assay in healthcare workers from University Hospitals of Shiraz, South West of Iran. Iran Red Crescent Med J 2013; 15: 477-482.

Talebi-Taher M, Javad-Moosavi SA, Entezari AH, et al. Comparing the performance of QuantiFERON-TB Gold and Mantoux test in detecting latent tuberculosis infection among Iranian health care workers. Int J Occup Med Environ Health 2011; 24: 359-366.

Thuong PH, Tam DB, Sakurada S, et al. Circulating granulysin levels in healthcare workers and latent tuberculosis infection estimated using interferon-gamma release assays. BMC Infect Dis 2016; 16: 580.

Topic RZ, Dodig S, Zoricic-Letoja I. Interferon-gamma and immunoglobulins in latent tuberculosis infection. Arch Med Res 2009; 40: 103-108.

Rie A, McCarthy K, Scott L, et al. Prevalence, risk factors and risk perception of tuberculosis infection among medical students and healthcare workers in Johannesburg, South Africa. S Afr Med J 2013; 103: 853-857.

Wei Z, Yang M, Quan B, et al. Prevalence of latent tuberculosis infection among healthcare workers in China as detected by two interferon-gamma release assays. J Hosp Infect 2013; 84: 323-325.

Whitaker JA, Mirtskhulava V, Kipiani M, et al. Prevalence and incidence of latent tuberculosis infection in Georgian healthcare workers. PLoS One 2013; 8: e58202.

Yalcin SS, Guler C. The influence of childhood BCG vaccination on tuberculin reactivity in adults with different risk factors. Trop Doct 2005; 35: 26-28.

Zhang X, Jia H, Liu F, et al. Prevalence and risk factors for latent tuberculosis infection among health care workers in China: a cross-sectional study. PLoS One 2013; 8: e66412.

Zhou F, Zhang L, Gao L, et al. Latent tuberculosis infection and occupational protection among health care workers in two types of public hospitals in China. PLoS One 2014; 9: 8 .

Zhu CT, Liu ZH, Li ZQ, et al. The performance and limitation of T-SPOT.TB for the diagnosis of TB in a high prevalence setting. $J$ Thorac Dis 2014; 6: 713-719.

Zwerling A, Joshi R, Kalantri SP, et al. Trajectories of tuberculosis-specific interferon-gamma release assay responses among medical and nursing students in rural India. J Epidemiol Glob Health 2013; 3: 105-117.

Do A, Limpakarnjarat K, Uthaivoravit W, et al. Increased risk of Mycobacterium tuberculosis infection related to the occupational exposures of health care workers in Chiang Rai, Thailand. Int J Tuberc Lung Dis 1999; 3: 377-381.

01 Keskiner R, Ergonul O, Demiroglu Z, et al. Risk of tuberculous infection among healthcare workers in a tertiary-care hospital in Ankara, Turkey. Infect Control Hosp Epidemiol 2004; 25: 1067-1071.

Naidoo S, Mahommed A. Knowledge, attitudes, behaviour and prevalence of TB infection among dentists in the western Cape. SADJ 2002; 57: 476-478.

Pai M, Gokhale K, Joshi R, et al. Mycobacterium tuberculosis infection in health care workers in rural India: comparison of a whole-blood interferon gamma assay with tuberculin skin testing. JAMA 2005; 293: 2746-2755. 
Roth V, Garrett D, Laserson K, et al. A multicenter evaluation of tuberculin skin test positivity and conversion among health care workers in Brazilian hospitals. Int J Tuberc Lung Dis 2005; 9: 1335-1342.

105 Yanai H, Limpakarnjanarat K, Uthaivoravit W, et al. Risk of Mycobacterium tuberculosis infection and disease among health care workers, Chiang Rai, Thailand. Int J Tuberc Lung Dis 2003; 7: 36-45.

106 Golchin M, Rostami M. Tuberculin test in nursing and human-sciences students. J Res Med Sci 2005; 10: $172-176$.

107 Kayanja HK, Debanne S, King C, et al. Tuberculosis infection among health care workers in Kampala, Uganda. Int J Tuberc Lung Dis 2005; 9: 686-688.

108 Levy MZ, Medeiros EAS, Shang N, et al. TST reversion in a BCG-revaccinated population of nursing and medical students, Sao Paulo, Brazil, 1997-2000. Int J Tuberc Lung Dis 2005; 9: 771-776.

109 Maciel ELN, Viana MC, Zeitoune RCG, et al. Prevalence and incidence of Mycobacterium tuberculosis infection in nursing students in Vitoria, Espirito Santo. Rev Soc Bras Med Trop 2005; 38: 469-472.

110 Silva V, Cunha A, Kritski A. Tuberculin skin test conversion among medical students at a teaching hospital in Rio de Janeiro, Brazil. Infect Control Hosp Epidemiol 2000; 23: 591-594.

111 Teixeira EG, Menzies D, Comstock GW, et al. Latent tuberculosis infection among undergraduate medical students in Rio de Janeiro State, Brazil. Int J Tuberc Lung Dis 2005; 9: 841-847.

112 Azimi S, Tebianian M, Mosavari N, et al. Evaluation of immunological parameters in purified protein derivative positive tuberculin workers. Iran J Basic Med Sci 2013; 16: 962-964.

113 Christopher DJ, James P, Daley P, et al. High annual risk of tuberculosis infection among nursing students in South India: a cohort study. PLoS One 2011; 6: e26199.

114 Cesur S, Hoca NT, Tarhan G, et al. [Evaluation of QuantiFERON-TB Gold In-Tube and tuberculin skin test in patients with tuberculosis, close contact of patients, health care workers and tuberculosis laboratory personnel.] Mikrobiyol Bul 2010; 44: 553-560.

115 Demir M, Tuncay E, Yenturk E, et al. [Increased risk of tuberculosis among health care workers in a chest diseases hospital.] Anatol J Clin Invest 2014; 8: 57-61.

116 Karaman S, Elif Ozturk C, Bahcebasi T. [Investigating of latent tuberculosis infection in hospital cleaning staff.] Duzce Med J 2011; 13: 32-44.

117 Ozturk N, Surucuoglu S, Ozkutuk N, et al. [Comparison of interferon-gamma whole blood assay with tuberculin skin test for the diagnosis of tuberculosis infection in tuberculosis contacts.] Mikrobiyol Bul 2007; 41: $193-202$.

118 Yilmaz I, Akcay S, Turkkan O, et al. [The frequency of asymptomatic atopy and relationship to latent tuberculosis in health workers.] Asim Allerji Immunoloji 2013; 11: 43-48.

119 Houben RM, Dodd PJ. The global burden of latent tuberculosis infection: a re-estimation using mathematical modelling. PLoS Med 2016; 13: e1002152.

120 Menzies D, Joshi R, Pai M. Risk of tuberculosis infection and disease associated with work in health care settings. Int J Tuberc Lung Dis 2007; 11: 593-605. 